\section{Apoptosis activation in human carious dentin. An immunohistochemical study}

\author{
C. Loreto, ${ }^{1}$ A. Psaila, ${ }^{1}$ G. Musumeci, ${ }^{1}$ \\ S. Castorina, ${ }^{1}$ R. Leonardi ${ }^{2}$ \\ 'Department of Biomedical and \\ Biotechnological Sciences, Human \\ Anatomy and Histology Section, \\ University of Catania \\ 2Department of General Surgery and \\ Medical-Surgical Specialities, University \\ of Catania, Italy
}

\begin{abstract}
The exact mechanisms and enzymes involved in caries progression are largely unclear. Apoptosis plays a key role in dentin remodelling related to damage repair; however, it is unclear whether apoptosis in decayed teeth is activated through the extrinsic or the intrinsic pathway. This ex vivo immunohistochemical study explored the localization of TRAIL, DR5, Bcl-2 and Bax, the main proteins involved in apoptosis, in teeth with advanced caries. To evaluate TRAIL, DR5, Bcl-2 and Bax immunoexpressions twelve permanent carious premolars were embedded in paraffin and processed for immunohistochemistry. The results showed that TRAIL and DR5 were overexpressed in dentin and in pulp vessels and mononuclear cells; strong Bax immunostaining was detected in dilated dentinal tubules close to the lesion, and Bcl-2 staining was weak in some dentin areas under the cavity or altogether absent. These findings suggest that both apoptosis pathways are activated in dental caries. Further studies are required to gain insights into its biomolecular mechanisms.
\end{abstract}

\section{Introduction}

Classically dental caries is defined as a multifactorial disease whose development requires a susceptible host, cariogenic bacteria, and a contribution from the diet; ${ }^{1}$ even if nowadays a new paradigm of dental caries has been postulated. This latter asserts that the caries process is the ubiquitous, natural metabolism in the biofilm that causes fluctuations in $\mathrm{pH}$. The interaction of this biofilm with the dental tissue may result in a carious lesion. ${ }^{2}$ The American Dental Association Council on Scientific Affairs has updated the definition of dental caries as the cumulative result of an imbalance of dynamic demineralization and remineralization process that causes a net mineral loss overtime. ${ }^{3}$ In particular, if there is an imbalance in the dynamic demineralization and remineralization process that takes place in the mouth, a mineral loss will be first noted at the enamel. As the process goes on, a cavity is formed and the dentin begins to demineralize, followed by the degradation of the dentin organic matrix. Even though the latter step is essential for caries progression, the exact mechanisms and enzymes involved are largely unclear. ${ }^{4-6}$

Tooth injuries and carious lesions activate repair mechanisms that rely on production of tertiary dentin by odontoblasts. Tertiary dentin has two forms, reparative and reactionary. Mild carious lesions induce production of reactionary, hypercalcified dentin, whereas advanced lesions lead to odontoblast apoptosis and production of reparative dentin by odontoblast-like cells differentiated from progenitors in the pulp. ${ }^{7}$

Apoptosis, or programmed cell death, is a genetically established form of cell control involved in tissue homeostasis. It plays a key role in tooth shape and size, tooth anomalies, and damage repair-related dentin remodelling. ${ }^{7,8-12}$ The process consists of two pathways: an extrinsic, or death receptor-mediated, and an intrinsic, or mitochondrial, pathway. ${ }^{13,14}$ The former pathway involves death receptors like Fas, tumour necrosis factor $\alpha$ (TNF- $\alpha$ ), and TNF$\alpha$-related apoptosis-inducing ligand (TRAIL). TRAIL is a type II transmembrane protein that induces apoptosis by binding to DR4 and DR5, its death domain-containing receptors (DR). DR5 recruits Fas-associated death domain (FADD) and caspase 8, leading to formation of a death-inducing signalling complex and to caspase 8 activation. ${ }^{15-17}$ Caspase 8 processing of its substrates (e.g., caspase-3, 6, and 9, or executioner caspases) results in chromatin condensation, nuclear fragmentation and cytoskeletal degradation. Activation of the executioner caspases makes the apoptotic process irreversible. The extrinsic and intrinsic pathways are activated respectively by exogenous and endogenous events, e.g. oxidative stress, ischaemia, and DNA damage. ${ }^{18} \mathrm{Bcl}-2$ protein family members play a key role in the mitochondrial pathway at the level of the outer mitochondrial membrane, allowing (pro-apoptotic action) or preventing (anti-apoptotic action) release of cytochrome $\mathrm{C}$ (CytC) from the inner mitochondrial lumen. Pro-apoptotic proteins include Bax, Bak or Noxa, Bid, and Bad, whereas Bcl-2 has an anti-apoptotic effect.
Correspondence: Prof. Carla Loreto, Department of Biomedical and Biotechnological Sciences, Section of Human Anatomy and Histology, University of Catania, Via S. Sofia 87, 95127 Catania, Italy.

Tel.+39.095.378 2038 - Fax: +39.095.378 2046.

E-mail: carla.loreto@unict.it

Key words: Apoptosis; TRAIL; DR5; Bax; Bcl-2; carious teeth.

Received for publication: 24 February 2015. Accepted for publication: 7 May 2015.

This work is licensed under a Creative Commons Attribution NonCommercial 3.0 License (CC BYNC 3.0).

(C) Copyright C. Loreto et al., 2015

Licensee PAGEPress, Italy

European Journal of Histochemistry 2015; 59:2513 doi:10.4081/ejh.2015.2513

By binding Bax, Bcl-2 acts as a competitor, preventing CytC release. CytC release thus leads to activation of apoptotic proteaseactivating factor-1 (APAF-1), which turns pro-caspase 9 into caspase 9 , whose dimerization leads in turn to formation of the apoptosome, a proteolytic executioner caspase 3-, 6- and 7-activating complex. Activation of the mitochondrial pathway may also result from the activation of caspase 8 , hence the extrinsic pathway; caspase 8 mediates the proteolytic cleavage of Bid to tBid, which inhibits Bcl-2 and permits CytC release.

Although it has been documented that apoptosis plays a pivotal role in dentin development and remodelling related to damage repair, how this pathophysiological process is activated is still far from clear. ${ }^{7}$ The present ex vivo study investigates apoptotic process activation in carious teeth to gain insights into its biomolecular mechanisms.

\section{Materials and Methods}

\section{Specimen collection}

Twelve permanent premolars (10 with gross caries and 2 sound teeth) were collected after their extraction at the Department of General Surgery and Medical-Surgical Specialities, University of Catania (Italy). Sample collection was approved by the Local Research Ethics Committee and informed written consent was obtained from each subject. The carious teeth were collected from patients requiring extrac- 
tion for various therapeutic reasons (ages 35$63)$. The diagnosis of carious lesions was carried out on colour and tactile criteria. Two healthy teeth obtained from extractions performed for orthodontic reasons were used as controls. Premolars from patients with clinically significant medical problems, teeth subjected to any form of endodontic therapy, and teeth with disease other than dental caries or with periapical disease suggesting pulp necrosis were excluded. Only fully erupted teeth were included in the study; all extractions, which were performed under local anaesthesia ( $2 \%$ lidocaine 1:80,000 epinephrine), were uncomplicated. After extraction teeth were immediately dewaxed in saline and fixed in $10 \%$ buffered formalin. The two sound teeth showed no colour change indicating caries within dentin; in those with gross chronic caries the colour change involved more than half of the dentin thickness. Caries depth was at stage 2.

\section{Hard dental tissue preparation for immunohistochemistry}

Two grooves perpendicular to the long tooth axis were cut with a bur equipped with an air-water spray. Teeth were divided into a top and a bottom half at the cementoenamel junction in order to study the crown dentin, fixed in 10\% neutral buffered formalin, and finally immersed in EDTA decalcification fluid (41.3 g disodium EDTA, $4.4 \mathrm{~g}$ $\mathrm{NaOH}$ in $1000 \mathrm{~mL}$ distilled water) for 6 weeks at $4^{\circ} \mathrm{C}$.

After an overnight wash, each tooth half was dehydrated in graded ethanols and embedded in paraffin with the anatomical orientation preserved. Sections $3-4 \mu \mathrm{m}$ in thickness were cut according to routine procedures, mounted on sialane-coated slides, and air-dried. Slides were subsequently dewaxed in xylene and hydrated using graded ethanols. fter rehydration endogenous peroxidase activity was quenched by treatment with $3 \% \mathrm{H}_{2} \mathrm{O}_{2}$ for $10 \mathrm{~min}$. Sections were treated $(3 \mathrm{x}$ $5 \mathrm{~min}$ ) in capped polypropylene slide-holders with citrate buffer ( $\mathrm{pH}$ 6.0) using a microwave oven $(750 \mathrm{~W})$, to unmask antigen sites. Nonspecific antibody binding was blocked by treatment with normal horse/goat serum diluted 1:20 in phosphate buffered saline (PBS) and $0.1 \%$ bovine serum albumin (Roche Applied Science, Penzberg, Germany).

The following primary antibodies were used: rabbit anti-human Bax polyclonal antibody (1:100); ready to use mouse anti-human Bcl-2 monoclonal antibody (both from Dako, Glostrup, Denmark); rabbit anti-TRAIL polyclonal antibody (1:100, Santa Cruz Biotechnology, Santa Cruz, CA, USA), and rabbit anti-DR5 polyclonal antibody (1:100, Novus Biologicals, Littleton, CO, USA). The primary
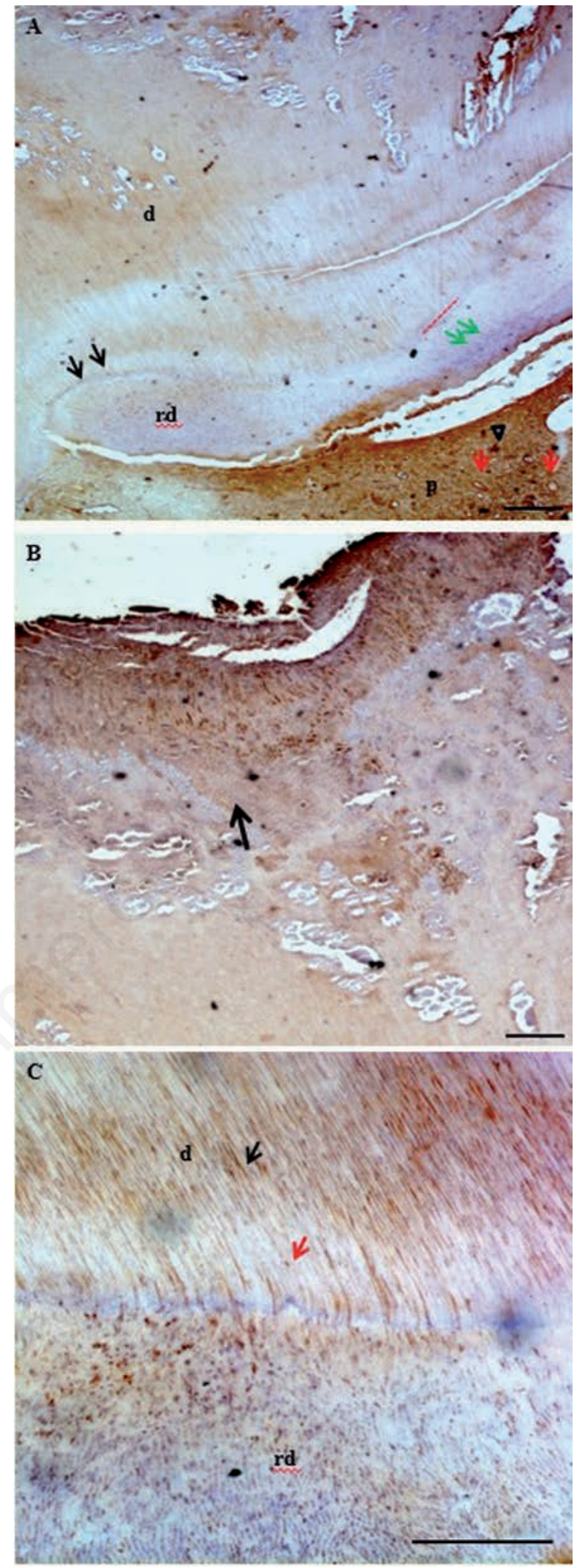

Figure 1. A) TRAIL immunoexpression in a carious tooth. TRAIL is overexpressed in secondary dentinal tubules (d), in odontoblasts sucked into tubules (black arrows) and in pulp (p), especially in vessels (red arrows) and mononuclear cells (arrowhead); the red dotted line indicates the boundary between reparative and secondary dentin and the green arrows the newly formed odontoblasts. rd: reparative dentin. B) TRAIL immunostaining in a carious tooth; the density of stained dentinal tubules was higher close to the bacterial localization (black arrow). C) TRAIL immunostaining in a carious tooth; TRAIL overexpression in dentinal tubules (black arrow) and odontoblasts sucked into the tubules (red arrow). d, secondary dentin; rd, reparative dentin; p, pulp. Scale bars: $100 \mu \mathrm{m}$. 


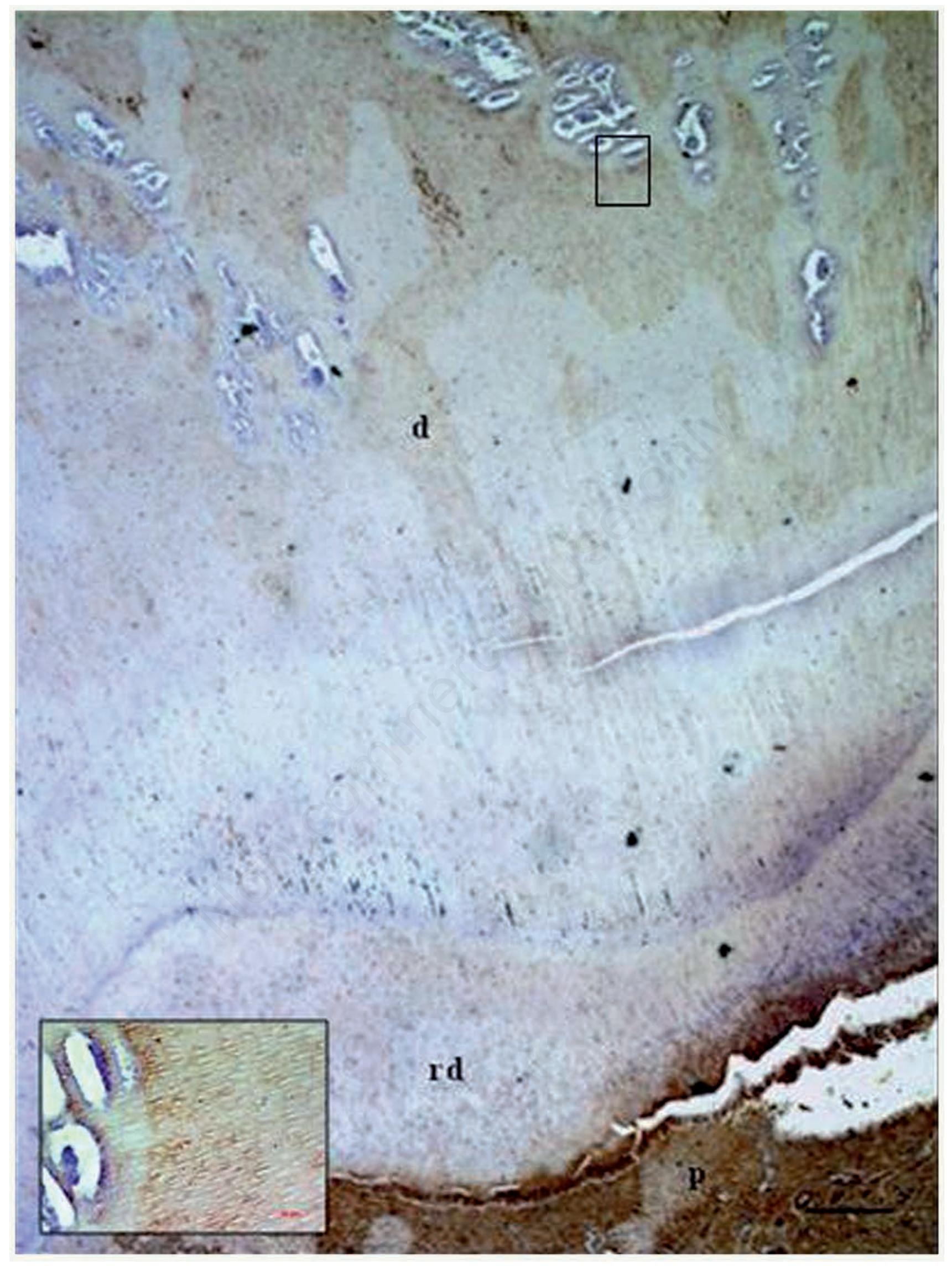

Figure 2. DR5 immunoexpression in a carious tooth in secondary dentinal tubules (d) and in pulp (p). rd, reparative dentin. Scale bar: $100 \mu \mathrm{m}$. Inset shows an higher magnification of DR5 immunoreaction in dentinal tubules. 


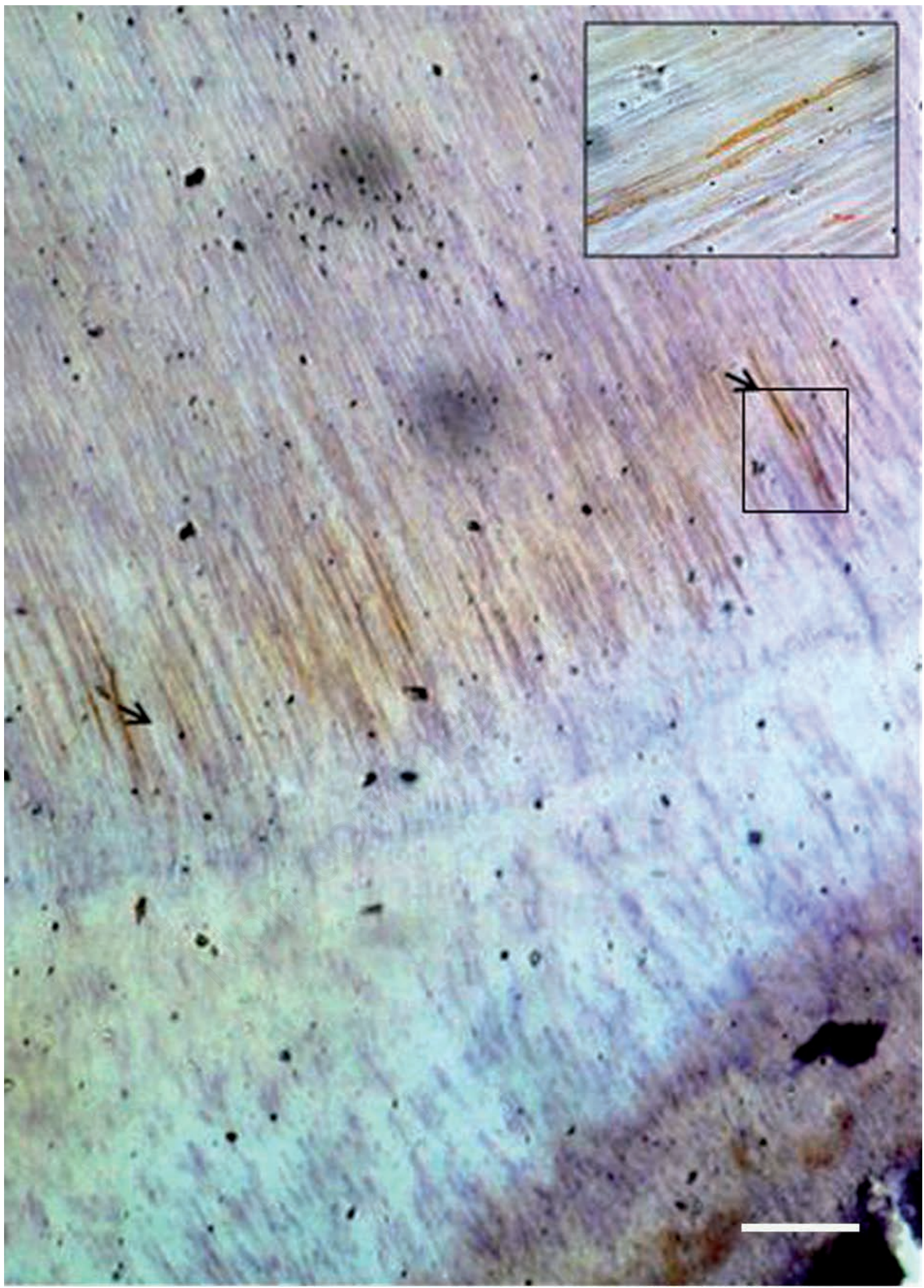

Figure 3. Bax immunoexpression in dilated dentinal tubules close to the caries lesion both in the peritubular zone and in Tomes' processes (plasma membrane and central area) (black arrows). Scale bar: $100 \mu \mathrm{m}$. Inset shows an higher magnification of Bax immunoreaction close to the caries lesion. 


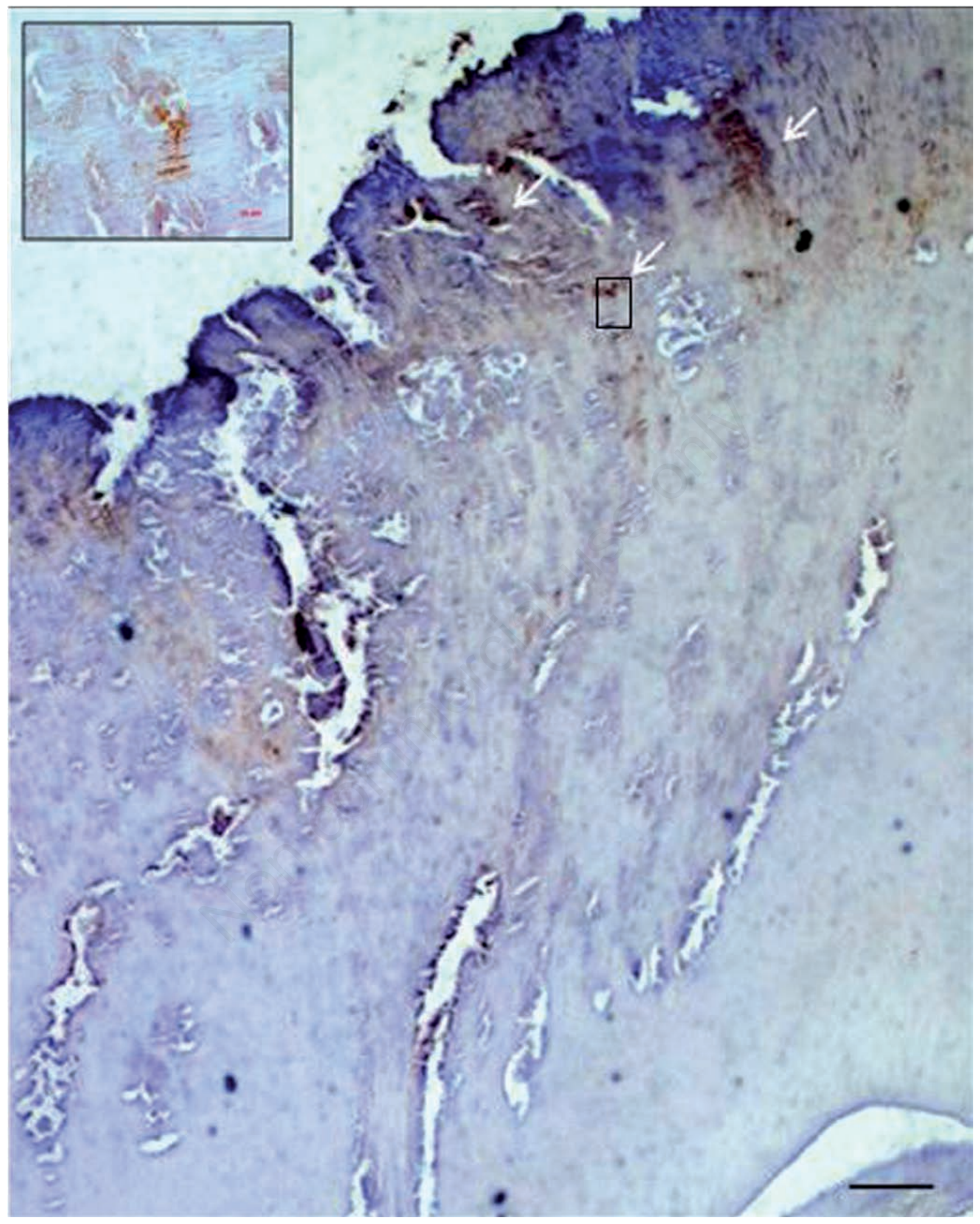

Figure 4. Weak Bcl-2 immunolabelling in some dentinal areas underlying the cavity (white arrows). Scale bar: $100 \mu \mathrm{m}$. Inset demonstrates Bcl-2 weak immunostaining in dentine underlying the cavity. 
antibodies were applied directly onto sections and slides were incubated overnight $\left(4^{\circ} \mathrm{C}\right)$ in a humid chamber. Sections were then washed in PBS, treated with a biotinylated antibody, and detected using peroxidaselabelled streptavidin, both incubated for 10 min at room temperature (LSAB+SystemHRP, Dako, Milan, Italy). The immunoreaction was assessed using a Zeiss Axioplan light microscope (Oberkochen, Germany) after incubating sections in $0.1 \% 3,3$ 'diaminobenzidine and $0.02 \%$ hydrogen peroxide solution (DAB substrate kit, Vector Laboratories, Burlingame, CA, USA) for 4 min. Sections were lightly counterstained with Mayer's haematoxylin then mounted in GVA mount (Zymed Laboratories, San Francisco, CA, USA). Positive controls were tissue specimens with known antigen positivity. Negative control sections were processed like the experimental slides, except that they were incubated with PBS instead of the primary antibody.

\section{Computerized image analysis}

Fifteen fields, area of $600.000 \mu^{2}$, randomly selected from each section, were analysed and the percent area stained with TRAIL, DR5, Bax and Bcl-2 antibodies was calculated using a software for image acquisition and histomorphometric analysis (AxioVision image analysis software Release 4.8.2 - SP2 SoftwareCarl Zeiss Microscopy Jena, Germany), which quantifies the area of positive immunolabelling in each field, expressed as \% positive, dark brown pixels of the analyzed fields. Digital micrographs were taken using the Axioplan light microscope (20x magnification) fitted with a digital camera (AxioCam MRc5, Carl Zeiss).

\section{Statistical analysis}

Data were analysed using the Student's $t$ test. Significance was set at $\mathrm{P}<0.05$ and $\mathrm{P}<0.01$. Mean and standard deviation were calculated. All data were analysed with SPSS program (SPSS ${ }^{\circledast}$ release 16.0, Chicago, IL, USA).

\section{Results}

TRAIL and DR5 immunostaining were detected only in dentin of carious teeth, where a very strong immunoreaction was observed close to the lesion. TRAIL overexpression involved especially the peritubular zone, Tomes' processes of secondary dentin below the lesion, and odontoblasts sucked into dentinal tubules (Figure 1A). The density of stained tubules was greater close to the bacterial localization (Figure 1B). Reparative dentin also showed some TRAIL immunoexpression (Figure 1C). TRAIL overexpression in pulp was detected especially in vessels and mononuclear cells (Figure 1A). DR5 staining showed a similar distribution and intensity as TRAIL (Figure 2). Bax overexpression was detected in dilated dentinal tubules close to the lesion both in the peritubular zone and in Tomes' processes (plasma membrane and central area) (Figure 3). Bcl-2 immunostaining was weak and was detected only in some dentin areas under the cavity (Figure 4 ). The immunohistochemical TRAIL and DR5 data

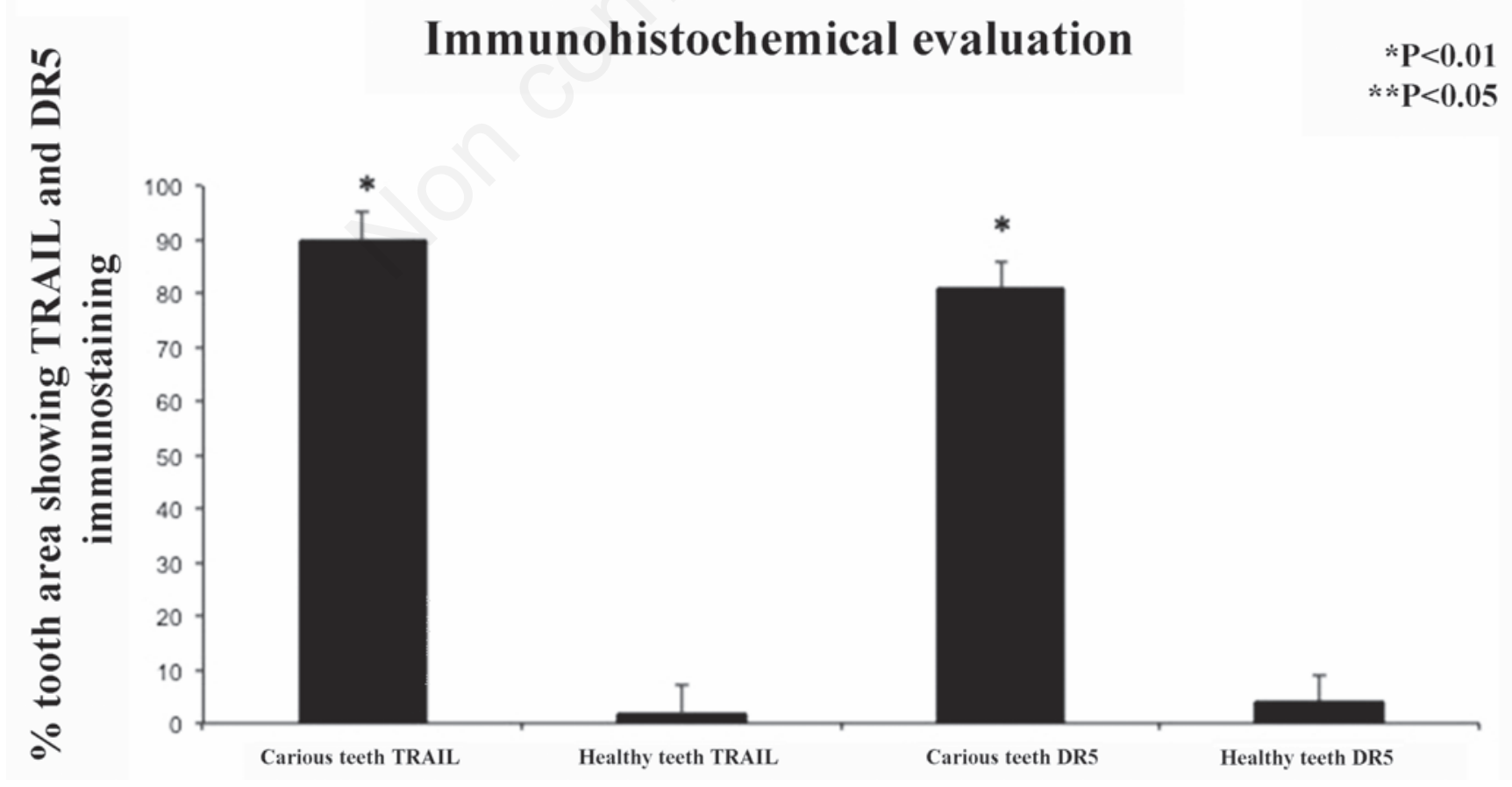

Figure 5. Histogram showing TRAIL and DR5 immunostaining evaluation. Percentage of tooth area showing TRAIL and DR5 immunostaining. Significance was set at $\mathrm{P}<0.05$ and $\mathrm{P}<0.01$. 
are illustrated in Figure 5 and those regarding Bax and Bcl-2 in Figure 6. Sound teeth were immunonegative. Immunoreactivity was not detected in the sections incubated with non-immune serum.

\section{Discussion}

Research into dentin carious tissue has demonstrated apoptotic odontoblasts using the TUNEL method and the immunohistochemical localization of caspase 3 (executive caspase), but the pathway activated by caries has never been investigated. ${ }^{7,19}$

Our study demonstrates activation of both apoptosis pathways by documenting TRAIL, DR5 and Bax overexpression and weak or absent Bcl-2 expression in secondary dentin, odontoblasts facing the carious lesion, and pulp cells found close to the injury site, in line with the data reported by Mitsiadis and coworkers. ${ }^{19}$ In fact bacterial infiltration and release of growth factors from dentin during demineralization induces odontoblast apoptosis at the lesion site and their replacement by a new generation of odontoblast-like cells derived from mesenchymal cells in the pulp. Dentin restoration and healing show similar- ities with embryonic tooth development; this also applies to cavities caused by mechanical damage. ${ }^{20}$

It has been documented that a new layer of reparative dentin forms from the outer side of the pulp chamber towards the chamber core in the area underlying the caries. Reparative dentin, a type of tertiary dentin, forms an irregular and less tubular structure than reactionary dentin, which is more similar to primary and secondary dentin. Reparative dentin often contains odontoblast-like cells that are similar to common bone osteocytes. In reparative dentinogenesis injury and disruption of the original odontoblasts activate growth factors, which eventually induce the differentiation of new odontoblasts from pulp stem cells. ${ }^{21-23}$ Dentinogenesis requires production of collagenous extracellular matrix, which undergoes mineralization. Dental pulp cells may also contribute to the mineralization. ${ }^{24}$ Although the dentin pulp is the main target of bacteria/toxins, odontoblasts seem to deposit protective reactionary dentin in response to dentin-solubilized regeneration signals. ${ }^{25,26}$

Dental caries and trauma generate an inflammatory response within the pulp that leads to accumulation of inflammatory cells, which in turn release inflammatory cytokines like TNF- $\alpha$. The present study also documented TRAIL expression. ${ }^{27,28}$ These insults stimulate pulp stem cells to differentiate into odontoblasts, which are responsible for reparative dentinogenesis by partly secreting dentin matrix proteins. $.^{29-31} \mathrm{Bcl}-2$ overexpression has been detected in odontoblasts immediately under a cavity preparation, suggesting that its elevation is an important mechanism for odontoblasts to manage injury and maintain their structural and functional integrity. ${ }^{7,32}$ Literature data demonstrate that $\mathrm{Bcl}-2$ promotes dentin damage repair by stemming the excess odontoblast apoptosis induced by mechanical injury. ${ }^{7}$ Mitsiadis and co-workers showed that the secretory activity of odontoblast is stimulated in teeth with carious lesions to produce either reparative or reactionary dentin, and documented apoptosis in odontoblasts facing the carious lesions. However, the mechanism activating the apoptotic process remains elusive. ${ }^{19}$ This suggests that Bcl-2 overexpression is needed to promote dentin repair, and that apoptosis is allowed by Bcl-2 inhibition via a higher expression of $\mathrm{tBid}$ and consequently of caspase $8{ }^{7}$ The weak Bcl-2 expression detected in our samples with advanced caries thus suggests that this tissue defence mechanism has failed to prevent the damage.

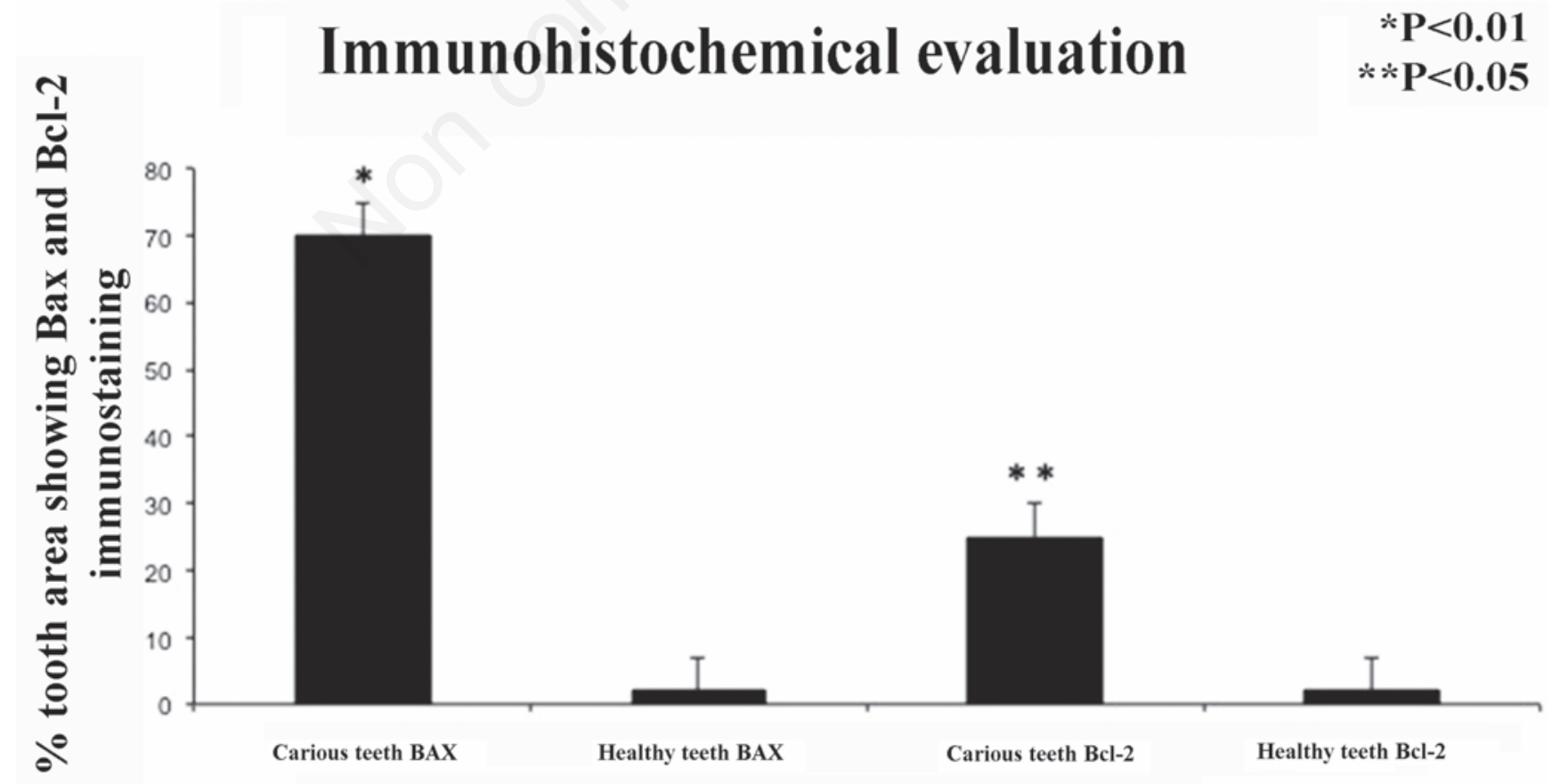

Figure 6. Histogram showing Bax and Bcl-2 immunostaining evaluation. Percentage of tooth area showing Bax and Bcl-2 immunostaining. Significance was set at $\mathrm{P}<0.05$ and $\mathrm{P}<0.01$. 
Additional in vitro studies, on odontoblast cultures, are clearly required to gain further insights into the modulation of the apoptotic mechanism by studying the activation and progression of the apoptotic pathways and their inhibitors allowing the identification of drugs that can influence this process.

\section{References}

1. Keyes PH. Recent advances in dental caries research bacteriology. Bacteriological findings and biological implications. Int Dent J 1962;12:443-64.

2. Kidd E. The implications of the new paradigm of dental caries. J Dent 2011;39 Suppl2:S3-8.

3. Young DA, Nový BB, Zeller GG, Hale R, Hart TC, Truelove EL, et al. The American Dental Association Caries Classification System for clinical practice: a report of the American Dental Association Council on Scientific Affairs. J Am Dent Assoc 2015; 146:79-86.

4. Loreto C, Galanti C, Musumeci G, Rusu MC, Leonardi R. Immunohistochemical analysis of matrix metalloproteinase-13 in human caries dentin. Eur $\mathrm{J}$ Histochem 2014;58:2318.

5. Martini D, Trirè A, Breschi L, Mazzoni A, Teti G, Falconi M, Ruggeri A Jr. Dentin matrix protein 1 and dentin sialophosphoprotein in human sound and carious teeth: an immunohistochemical and colorimetric assay. Eur J Histochem 2013;57:e32

6. Katz S, Park KK, Palenik CJ. In-vitro root surface caries studies. J Oral Med 1987;42:40-8.

7. Zhang W, Ju J. Odontoblast-targeted Bcl-2 overexpression promotes dentin damage repair. Arch Oral Biol 2012;57:285-92.

8. Vaahtokari A, Åberg T, Thesleff I. Apoptosis in the developing tooth: association with an embryonic signalling center and suppression by EGF and FGF-4. Development 1996;122:121-9.

9. Kim JY, Cha YG, Cho SW, Kim EJ, Lee MJ, Lee JM, Cai J, Ohshima H, Jung HS. Inhibition of apoptosis in early tooth development alters tooth shape and size. J Dent Res 2006;85:530-5.
10. Lesot H, Vonesch JL, Peterka M, Tureckova J, Peterkova R, Ruch JV. Mouse molar morphogenesis revisited by three-dimensional reconstruction. II. Spatial distribution of mitoes and apoptosis in cap to bell staged first and second upper molar teeth. Int $\mathbf{J}$ Dev Biol 1996;40:1017-31.

11. Viriot L, Peterkova R, Vonescch JL, Peterka M, Ruch JV, Lesot H. Mouse molar morphogenesis revisited by three-dimensional reconstruction. III. Spatial distribution of mitoses and apoptoses up to bell-staged first lower molar teeth. Int J Dev Biol 1997;41:679-90.

12. Sharma PK, Kharbanda OP. Role of programmed cell death in dental anomalies associated with cleft lip and palate. Med Hypotees 1991;41:679-90.

13. Bhardwaj A, Aggarwal BB. Receptor-mediated coreography of life and death. J Clin Immunol 2003;23:317-32.

14. Chaudari BR, Murphy RF, Agrawal DK. Following the TRAIL to apoptosis. Immunol Res 2006;35:249-62.

15. Baetu TM, Hiscott J. On the TRAIL to apoptosis. Cytokine Growth Factor Rev 2002;13: 199-207.

16. Falschlehner C, Emmerich $\mathrm{CH}$, Gerlach B, Walczak H. TRAIL signaling: decisions between life and death. Int J Biochem Cell Biol 2007;39:1462-75.

17. Chen B, Ma B, Yang S, Xing X, Gu R, Hu Y. DR5 and DcR2 are expressed in human lumbar intervertebral discs. Spine (Phila Pa 1976) 2009;34:E677-81.

18. Huppertz B, Kaufmann HGFP. The apoptosis cascade-morphological and immunohistochemical methods for its visualization. Anat Embryol 1999;200:1-18.

19. Mitsiadis TA, De Bari Cm About I. Apoptosis in developmental and repairrelated human tooth remodelling: a view from the inside. Exp Cell Res 2008;314: 869-77.

20. Smith AJ, Lesot H. Induction and regulation of crown dentinogenesis: embryonic events as a template for dental tissue repair? Crit Rev Oral Biol Med 2001;12: 425-37.

21. Tziafas D. Dentinogenic potential of the dental pulp: facts and hypotheses. Endodontic Topics 2007;17:42-64.

22. Orsini G, Majorana A, Mazzoni A, Putignano A, Falconi M, Polimeni A, et al.
Immunocytochemical detection of dentin matrix proteins in primary teeth from patients with dentinogenesis imperfecta associated with osteogenesis imperfecta. Eur J Histochem 2014;58:2405.

23. Horst OV, Horst JA, Samudrala R, Dale BA. Caries induced cytokine network in the odontoblast layer of human teeth. BMC Immunol 2011;24:12:9

24. Butler WT, Brunn JC, Qin C. Dentin extracellular matrix (ECM) proteins: comparison to bone ECM and contribution to dynamics of dentinogenesis. Connect Tissue Res 2003;44:171-78.

25. Chmilewsky F, Jeanneau C, Laurent $P$, Kirschfink M, About I. Pulp progenitor cell recruitment is selectively guided by a $\mathrm{C} 5 \mathrm{a}$ gradient. J Dent Res 2013;92:532-9.

26. Teti G, Salvatore V, Ruggeri A, Manzoli L, Gesi M, Orsini G, et al. In vitro reparative dentin: a biochemical and morphological study. Eur J Histochem 2013;57:e23.

27. Pezelj-Ribaric S, Anic I, Brekalo I, Miletic I, Hasan M, Simunovic-Soskic M. Detection of tumor necrosis factor alpha in normal and inflamed human dental pulps. Arch Med Res 2002;33:482-484.

28. Bletsa A, Heyeraas KJ, Haug SR, Berggreen E. IL-1 alpha and TNFalpha expression in rat periapical lesions and dental pulp after unilateral sympathectomy. Neuroimmunomodulation 2004;11: 376-84.

29. Butler WT. Dentin matrix proteins and dentinogenesis. Connect Tissue Res 1995;33:59-65.

30. Massa FL, Ramachandran A, George A, Arana-Chavez VE. Developmental appearance of dentin matrix protein 1 during the early dentinogenesis in rat molars as identified by high resolution immunocytochemistry. Histochem Cell Biol 2005;124: 197-205.

31. Martinez EF. Herdy Da Silva LA, Furuse C, Soares De Araujo N, Cavalcanti De Araujo V. Dentin matrix protein 1(DMP1) espression in developing human teeth. Braz Dent J 2009;20:365-9.

32. Kitamura C, Kimura K, Nakayama T, Toyoshima K, Terashita M. Primary and secondary induction of apoptosis in odontoblasts after cavity preparation of rat molars. J Dent Res 2001;80:1530-4. 